\title{
Rainfall as a driver of epidemic cholera: comparative model assessments of the effect of intra-seasonal precipitation events
}

\author{
Joseph Lemaitre ${ }^{\mathrm{a}}$, Damiano Pasetto ${ }^{\mathrm{a}}$, Javier Perez-Saez ${ }^{\mathrm{a}}$, Carla Sciarra ${ }^{\mathrm{b}}$, \\ Joseph Francis Wamala ${ }^{\mathrm{c}}$, Andrea Rinaldo, ${ }^{\mathrm{a}, \mathrm{d}, *}$ \\ ${ }^{a}$ Laboratory of Ecohydrology, École Polytechnique Fédérale de Lausanne (EPFL), 1015 \\ Lausanne $(\mathrm{CH})$ \\ ${ }^{b}$ Dipartimento di Ingegneria dell'Ambiente, del Territorio e delle Infrastrutture, \\ Politecnico di Torino, Corso Duca degli Abruzzi, 24, 10129 Torino, (IT) \\ ${ }^{c}$ WHO South Sudan \\ ${ }^{d}$ Dipartimento ICEA, Università di Padova, 35100 Padova (IT)
}

\section{Abstract}

The correlation between cholera epidemics and climatic drivers, in particular seasonal tropical rainfall, has been studied in a variety of contexts owing to its documented relevance. Several mechanistic models of cholera transmission have included rainfall as a driver by focusing on two possible transmission pathways: either by increasing exposure to contaminated water (e.g. due to worsening sanitary conditions during water excess), or water contamination by freshly excreted bacteria (e.g. due to washout of open-air defecation sites or overflows). Our study assesses the explanatory power of these different modeling structures by formal model comparison using deterministic and stochastic models of the type susceptible-infected-recovered-bacteria (SIRB). The incorporation of rainfall effects is generalized using a nonlinear function that can increase or decrease the relative importance of the large precipitation events. Our modelling framework is tested against the daily

*to whom correspondence should be sent

Preptintuisubdditteste jostephr.dymaitre@epfl.ch (Joseph Lemaitre), November 3, 2018 damiano.pasetto@epfl.ch (Damiano Pasetto), javier.perezsaez@epfl.ch (Javier Perez-Saez), carla.sciarra@polito.it (Carla Sciarra), walamaj@who.int (Joseph Francis Wamala), andrea.rinaldo@epfl.ch (Andrea Rinaldo)

$U R L:$ https://echo.epfl.ch/ (Andrea Rinaldo) 
epidemiological data collected during the 2015 cholera outbreak within the urban context of Juba, South Sudan. This epidemic is characterized by a particular intra-seasonal double peak on the incidence in apparent relation with particularly strong rainfall events. Our results show that rainfall-based models in both their deterministic and stochastic formulations outperform models that do not account for rainfall. In fact, classical SIRB models are not able to reproduce the second epidemiological peak, thus suggesting that it was rainfall-driven. Moreover we found stronger support across model types for rainfall acting on increased exposure rather than on exacerbated water contamination. Although these results are context-specific, they stress the importance of a systematic and comprehensive appraisal of transmission pathways and their environmental forcings when embarking in the modelling of epidemic cholera.

Keywords: epidemiological drivers, waterborne disease epidemics, Juba, South Sudan, environmental exposure

\section{Introduction}

Two main exposure pathways fuel cholera transmission across endemic and epidemic settings. First, as famously discovered by John Snow during the 1854 London cholera outbreak, an indirect exposure occurs from consumption of unsafe water contaminated by raw sewage [1]. Here, rainfall and the ensuing hydrologic transport processes play a major role in water contamination, for instance through the washout of open-air defecation sites and raw sewage circulation in the environment which is thought to have caused the revamping of the Haitian 2010 outbreak [2]. Direct, or human-to-human 
exposure occurs when the bacteria is transmitted from an infected directly to a healthy person, for example via contaminated food. In this case, environmental factors do not play a major role, except for possibly enhanced transmission due to (over-)crowding [3, 4]. It is known that the combination of environmentally-mediated and direct exposures shapes the spatio-temporal distribution of cases during cholera epidemics $[5,6,7,8]$.

The importance of climatic and environmental factors in the transmission of cholera, namely temperature and rainfall, has been highlighted across settings. Indeed, the relationship between cholera and climate has long been studied, moving from seminal works linking cholera outbreaks to anomalies in the El Niño Southern Oscillation $[9,10]$ that have paved the way for a new field in epidemiological research. For large-scale infection patterns, many studies highlighted the role of climatic drivers on cholera dynamics, mostly focusing on climate change effects on disease spread [11, 12, 13, 14, 15, 16, 8] or on the impacts of spatial and temporal heterogeneities [17, 18, 19, 20, 21, $22,23]$. While the effect of temperature on cholera transmission has been well unravelled, mainly regarding bacterial biology and ecology in natural environments, that of rainfall remains to be fully elucidated, possibly due to the multiple ways in which it can influence transmission at the local and regional scales $[2,24,25]$. Indeed, intense rainfall events have been shown to alter infection risk through a variety of potential mechanisms, including: flooding, leading to raw sewage contamination of water sources [26, 11]; increased hydrologic transport-driven iron availability in environmental waters that enhances pathogen survival and the expression of toxins [27, 28, 29]; dry spells inducing persistent low water levels leading to increased use of unsafe 
water sources [30]; and crowding during strong flood events [17].

Most countries where associations between rainfall and cholera risk have been studied experience endemic cholera transmission [8]. Empirical studies have shown a range of correlations, both positive and negative, endowed with time lags ranging from weeks to months [26, 31, 12]. In general, rainfall has been found to enhance cholera transmission, but there is evidence that propagation buffer effects in wet regions may be due to pathogen dilution [26]. Such variability reflects the variety of potential mechanisms whereby rainfall may alter infection risk. Similarly, a clear empirical correlation between intense rainfall and enhanced transmission is found in several regions hit by cholera epidemics $[32,30,33,34,35]$. The Haitian case, which has been in the midst of a major outbreak since October 2010 [36, 35, 37, 38, 39, 40, 41], has been studied under that angle, but its patterns have been argued to require a specific understanding [42]. Rainfall therein is empirically known to be directly associated with sudden resurgence of cholera infections via the analysis of reported cases [35], but a direct, causal relationship has only begun to be quantitatively examined $[2,24,43]$. Indeed, cholera case counts tend to rise sharply at the onset of seasonal heavy rains $[44,45,46]$. Notably, for the Haitian outbreak, such nexus has been addressed theoretically [2, 24]. Results therein showed that at all spatial scales and locations examined, the tropical storms were significantly correlated with increased cholera incidence with lags of the order of a few days. As a consequence, accounting for the related forcing of dynamic models resulted in improved fits of reported incidence.

Properly incorporating the effects of rainfall in mathematical models of 
cholera transmission is thus paramount to discriminate among the abovementioned alternative transmission pathways, thus unlocking a predictive framework to evaluate the potentially rainfall-sensitive efficacy of available intervention strategies in endemic and epidemic settings including vaccination, antibiotics, and improved access to water sanitation and hygiene (WaSH), leveraging the numerous solutions that exist for rainfall forecasting $[2,43]$. This becomes critically important when evaluating the number of averted infections by deploying vaccines, as was done in the aftermath of the passage of Hurricane Matthew [40], or considering optimal deployment in space and time.

Rainfall has been accounted for in two main fashions in recent mathematical models of cholera. On one side, a contamination-centered approach suggesting that bursts of infections could be linked to increased contamination of the water compartment [2]. This process conceptualizes the washout of open-air defecation sites by hydrologic transport. The same 'transport' effect may be realized by sewer collectors' overflows. In fact, both mechanisms have the net effect of charging progressively the bacterial concentration in the water reservoir [47]. Pathogens' loads are washed out from a hydrologic catchment enclosing human settlements and their infective individuals shedding bacteria. Therein, pathogen survival and thus the toxicity of their loads depend on hydrologic residence time distributions $[2,8]$. Such loads increase as a function of rainfall, which acts as proxy of runoff volumes. The second approach is exposure-centered and employs a rainfall-dependent exposure rate subsuming both pathogen availability and the probability of the ingestion of contaminated water during wet spells [24]. Although both ap- 
proaches are physically plausible, they have not been compared directly on the same datasets within a formal statistical framework, which would allow to highlight their respective merits and further recommendations for their use in different settings.

Here, we compare the explanatory power of these different types of rainfalldriven mechanistic models applied to a cholera outbreak in South Sudan. We quantitatively examine the link between rainfall and cholera during the outbreak recorded in Juba in 2015, when an intra-seasonal peak of cholera cases was recorded possibly in correspondence to intense precipitation events. The analysis of the lagged relationship between rainfall rates and revamped cholera incidence is addressed via dynamical compartmental models considered both in deterministic and stochastic versions incorporating direct (human-to-human) and indirect (water-to-human) disease transmission, and rainfall effects on contamination and exposure.

This paper is organized as follows. The Materials and Methods section introduces to the data sets employed in our exercises and the general modeling rationale and framework. Results and a discussion follow, highlighting the role of rainfall in this specific case study with a view to the systematic comparative analysis of the importance of intra-seasonal precipitation events in epidemic and endemic cholera in other settings. A set of conclusions building on the modelling results and suggesting the way forward closes then the paper. 


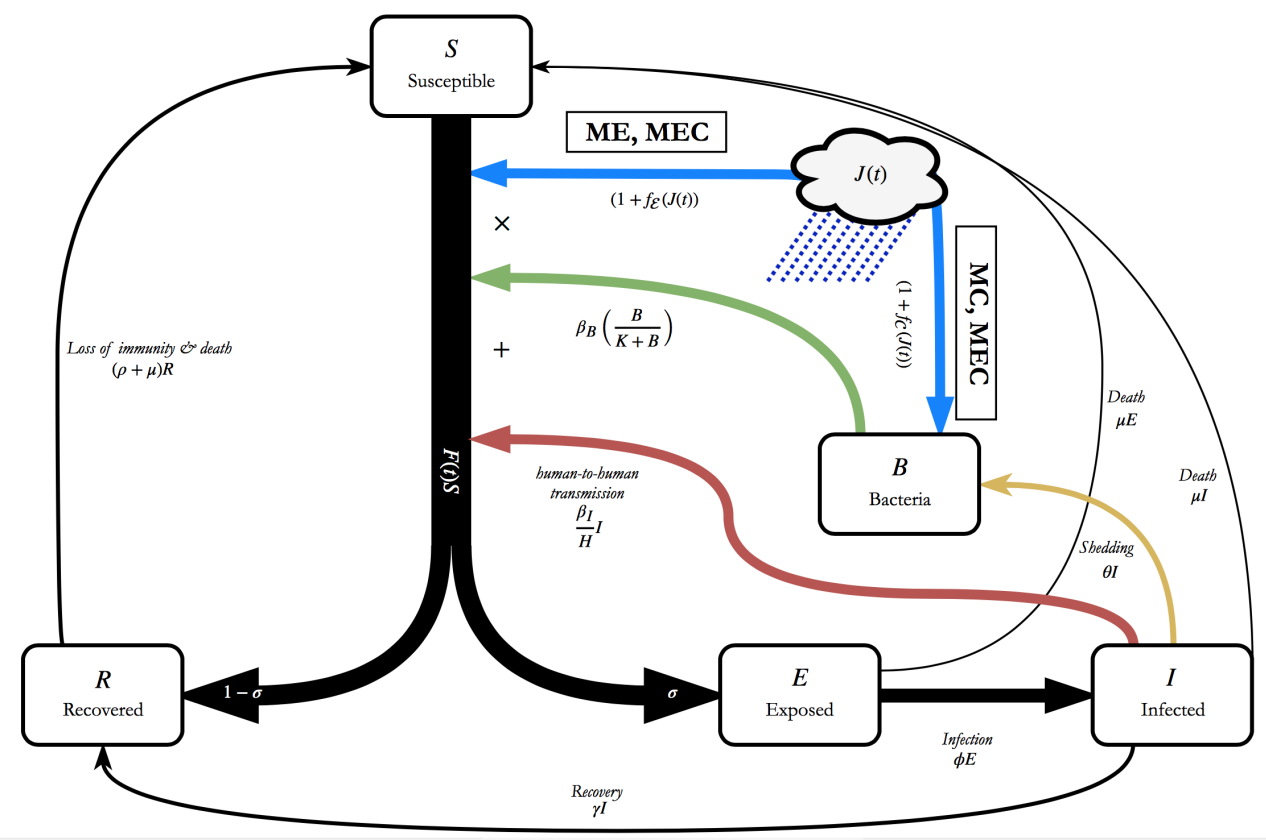

Figure 1: Flow diagram for our cholera models, with the different variations $\mathbf{M E}, \mathbf{M R}$, and MEC indicated. 


\section{Materials and methods}

We base our analysis of the cholera outbreak in Juba on a general epidemiological model that encompasses previous approaches to account for the effect of rainfall on cholera transmission. The proposed model builds on the classic susceptible $S$, infected $I$, and recovered $R$ compartments for individuals, with an additional variable $B$ describing the concentration of the bacteria in the environment (thus, the model is named SIRB). Previous modelling exercises had considered rainfall intensity $J(t)$ either to i) multiplicatively increase water contamination with bacteria shed by infected individulas $[43,40]$, or ii) assumed that the rainfall multiplicatively increases the exposure to contaminated water [24] (details on these two modelling frameworks are the Supplementary Information, SI, Section S.1). Aiming at a systematic comparison of the effect of rainfall through these two different transmission pathways, we here consider a generalized formulation of these cholera-forced models, wherein both formulations are nested.

Given the daily temporal resolution at which incidence data was available for the 2015 Juba's epidemic (see Section 3), we here introduce in addition to the S-I-R-B variables, a compartment of exposed individuals $E$, to describe the incubation period of the disease (from $12 \mathrm{~h}$ to 5 days [48]). This compartment is necessary to account the lag between the time of infection and the onset of the symptoms which result in reported cases. Moreover, in order to account for the vaccination campaigns that were deployed in Juba during August 2015, four compartments $\left(V^{S}, V^{E}, V^{I}\right.$, and $\left.V^{R}\right)$ are added to describe the dynamics of vaccinated individuals and their removal from the pool of susceptibles. 
The proposed generalized cholera model is described in Figure 1, and formulated as:

$$
\begin{aligned}
\frac{d E}{d t} & =\sigma F(t) S-(\phi+\mu+\nu) E \\
\frac{d I}{d t} & =\phi E-(\gamma+\mu+\alpha) I \\
\frac{d R}{d t} & =(1-\sigma) F(t) S+\gamma I-(\rho+\mu+\nu) R \\
\frac{d B}{d t} & =-\mu_{B} B+\theta\left[1+f_{\mathcal{C}}(J(t))\right]\left(I+V^{I}\right) \\
\frac{d V^{S}}{d t} & =\nu S-\mu V^{S}+\rho_{v} V^{R}-(1-\eta) F(t) V^{S} \\
\frac{d V^{E}}{d t} & =\nu E+\sigma(1-\eta) F(t) V^{S}-(\phi+\mu) V^{E} \\
\frac{d V^{I}}{d t} & =\phi V^{E}-(\gamma+\alpha+\mu) V^{I} \\
\frac{d V^{R}}{d t} & =\nu R-\left(\mu+\rho_{v}\right) V^{R}+\gamma V^{I}+(1-\sigma)(1-\eta) F(t) V^{S}
\end{aligned}
$$

where $F(t)$ takes into account both human-to-human transmission and nonlinear water-to-human transmission:

$$
F(t)=\beta_{B}\left[\frac{B}{K+B}\right]\left(1+f_{\mathcal{E}}(J(t))\right)+\frac{\beta_{I}}{H}\left(I+V^{I}\right) .
$$

As few reliable data on changes in Juba's population are available for the years of interest (2014 and 2015), the total population $H$, is assumed to be constant, which implies that the number of susceptible individuals at time $t$ is $S(t)=H-I(t)-E(t)-R(t)-V^{S}(t)-V^{E}(t)-V^{I}(t)-V^{R}(t)$. Individuals are removed from the susceptible compartment $S$ at rate $F(t)$, becoming either symptomatically or asymptomatically infected with probabilities $\sigma$ and $(1-\sigma)$, respectively. Symptomatically infected, $I$, shed $V$. cholerae into the local watershed at rate $\theta$. The infectious period is governed by parameter $\gamma$, 
which determines the portion of infected individuals that enter the recovered compartment $R$, joining the asymptomatic infected. Recovered individuals return to the susceptible compartment at a rate $\rho$, describing the average rate of loss of immunity for individuals that previously had been asymptomatic or symptomatic infected. Parameters $\alpha$ and $\mu$ concern cholera related and unrelated death rates, respectively. The compartment $B$ quantifies the concentration of $V$. cholerae in the local (conceptualized) water reservoir, which is used to estimate the probability of exposure to the contaminated water in (9) through the term $\beta_{B} \frac{B}{K+B}$, where $\beta_{B}$ is the maximum exposure rate and $K$ is the half-saturation constant of the dose-response function of $V$. cholerae [47]. Parameter $K$ is usually set to one by considering the change of variable $\tilde{B}=B / K$. The parameter $\mu_{B}$ expresses the rate of decay of bacteria in the environment. Exposed individuals become symptomatic infected at a rate $\phi$, which corresponds to an average incubation period of $1 / \phi \approx$ 1.5 days [48]. Functions $f_{\mathcal{C}}(J(t))$ and $f_{\mathcal{E}}(J(t))$ account for the rainfall effect respectively by increasing the bacteria contamination in the water reservoir (as the term $\lambda J(t)$ in eq. S.3) or directly through amplifying the exposure in the force of infection (as the term $\lambda J(t)$ in eq. S.8).

With the objective of assessing the importance of rainfall on cholera transmission, we here propose a generalization of the linear relation in eqs. (S.3) and (S.8) by using a nonlinear function form for $f_{\mathcal{C}, \mathcal{E}}(J(t))$, reading:

$$
f_{\mathcal{C}, \mathcal{E}}(J(t))=\lambda_{\mathcal{C}, \mathcal{E}}\left(\frac{J(t)}{\max _{t} J(t)}\right)^{\alpha_{\mathcal{C}, \mathcal{E}}}
$$

where the subscripts $\mathcal{C}, \mathcal{E}$ respectively denote the effect of rainfall on exposure and contamination, $\max _{t} J(t)$ is the maximum recorded rainfall intensity during the epidemic, and the $\alpha_{\mathcal{C}, \mathcal{E}} \geq 0$ controls for the relative importance of 
different rainfall intensities in their effect on the force of infection. Indeed, since the ratio $\frac{J(t)}{\max _{t} J(t)} \in[0,1]$, for $\alpha_{\mathcal{C}, \mathcal{E}} \gg 1$ the ratio will tend to 0 for all small precipitation events, leaving only the effect of the strongest events, whereas for $\alpha_{\mathcal{C}, \mathcal{E}}<1$ all precipitation events will be assigned a similar weight in the FOI. We also note that by setting $\alpha_{\mathcal{C}, \mathcal{E}}=1$ we recover the formulations in eqs. (S.3) and (S.8). The flexibility allowed by (10) is therefore able to discriminate between rainfall effects along a continuum from acting on disease transmission regardless of intensity to a threshold-like effect for the largest events which could be associated to severe flooding causing damages to the city's water and sanitary system, for instance leading to sewer overflow. Note that in (9) precipitation enters in the term $\left(1+f_{E}(J(t))\right)$ which entails water-to-human transmission also when $J(t)=0$ (differently from what happens in eq. S.8).

During the vaccination campaign, OCV doses are assumed to be distributed with equal rate $\nu$ to susceptible, exposed and recovered individuals, which enter the compartments $V^{S}, V^{E}$ and $V^{R}$. As the OCV provides only a partial immunity having efficacy $\eta, 0 \leq \eta \leq 1$, vaccinated susceptibles $\left(V^{S}\right)$ can become exposed $\left(V^{E}\right)$ through a decreased force of infection of a factor $(1-\eta)$ with respect to non-vaccinated individuals. Vaccinated infected individuals behave exactly like infected ones, but are placed in a different compartment to exclude them from future vaccination campaigns. After recovering at rate $\gamma$, they lose their vaccine protection at rate $\rho_{v}$.

\subsection{Model comparison}

Here we assess the relevance of the two rainfall-driven transmission pathways by comparing the impact the results of the following models: 
MN SIRB model without rainfall: $\lambda_{\mathcal{C}}=\lambda_{\mathcal{E}}=0, \beta_{I}=0$, as the null hypothesis for the importance of rainfall.

MC SIRB model using the approach described in eqs. (S.1-S.4) accounting for rainfall enhances the contamination of the water reservoir $[2,43]$ : $\lambda_{\mathcal{E}}=0, \beta_{I}=0$.

ME SIRB model using the formulation rainfall as in eqs. (S.5-S.8) where rainfall increases the exposure to bacteria [24]: $\lambda_{\mathcal{C}}=0, \beta_{I}=0$.

MEC SIRB model combining both approaches MC and ME $\left(\beta_{I}=0\right)$. Both ways of accounting rainfall play a role simultaneously.

For each model we explore the possibility of adding explicitly human-tohuman transmission $\left(\beta_{I}>0\right)$, which is indicated with an $\mathbf{H}$ at the end of the model name: MNH, MCH, MEH, and MECH. Table 1 summarizes the different parameters associated with the considered models.

The results of the 8 models that arise from this setting are compared on the basis of their ability to match the time series of daily reported cases during the cholera epidemic in Juba of 2015 (see Section 3). Table S.1 summarizes which parameters are calibrated for each model and their prior distribution. The degrees of freedom of the models, $n_{p}$, vary from $n_{p}=7$ for MN to $n_{p}=12$ for MECH. Given the low number of daily reported cases and their ensuing variability, we also implement a stochastic equivalent of the deterministic ODE system (1-8) formulated as a continuous time partially observed Markov process model, accounting for both demographic and disease transmission stochasticities [49] (details Section S.2). 


\begin{tabular}{lccccc}
\hline Model & $\lambda_{\mathcal{C}}$ & $\alpha_{\mathcal{C}}$ & $\lambda_{\mathcal{E}}$ & $\alpha_{\mathcal{E}}$ & $\beta_{I}$ \\
\hline MN & - & - & - & - & - \\
MNH & - & - & - & - & $\mathrm{X}$ \\
MC & $\mathrm{X}$ & $\mathrm{X}$ & - & - & - \\
ME & - & - & $\mathrm{X}$ & $\mathrm{X}$ & - \\
MCH & $\mathrm{X}$ & $\mathrm{X}$ & - & - & $\mathrm{X}$ \\
MEH & - & - & $\mathrm{X}$ & $\mathrm{X}$ & $\mathrm{X}$ \\
MEC & $\mathrm{X}$ & $\mathrm{X}$ & $\mathrm{X}$ & $\mathrm{X}$ & - \\
MECH & $\mathrm{X}$ & $\mathrm{X}$ & $\mathrm{X}$ & $\mathrm{X}$ & $\mathrm{X}$ \\
\hline
\end{tabular}

Table 1: Parameters considered in the eight compared models. $\lambda$ and $\alpha$ characterize the functional forms considering the precipitation (eq. 10). $\beta_{I}$ is the exposure for human-tohuman transmission.

Calibration of the deterministic model is performed using a Markov Chain Monte Carlo (MCMC)-based algorithm, which draws samples from the posterior distribution of the parameters. Inference on the stochastic model is performed using a frequentist multiple iterated filtering algorithm. Both model were fit against the daily reported cases accounting for over- or underreporting, and assuming a Poisson distribution. Models were then compared using the Bayesian Information criterion (BIC), Bayes factors, and the likelihood ratio test for the nested models (details given in Sections S.3 and S.4). 


\section{Case study: the 2015 Juba's outbreak}

In the past years, South Sudan had been struck by several cholera outbreaks (details in S.5). Here we focus on the analysis of the outbreak in Juba during 2015, when a particular double peak of cholera cases occurred, probably associated to a strong intra-seasonal precipitation event (Figure 2). Epidemiological records for the 2014 and 2015 cholera epidemics included cholera cases and hospitalization time series at the second-lowest administrative level (named Payams in South Sudan)as reported by SSMoH $[50,51,52]$. The recorded cases in the 7 Payams that constitute the administrative area of Juba have been aggregated to obtain the reported time series for the county level. We assigned the population values for Juba county as in the official projections provided by the South Sudan National Bureau of Statistics [53]. These values were validated against the growth rate value stated by CIA [54]. Daily rainfall estimates $\left[\mathrm{mm} \mathrm{d}^{-1}\right]$ were downloaded from the Climate Data Library (National Oceanic and Atmospheric Administration, NOAA) [55] for the years 2014 and 2015, with spatial resolution of $0.1^{\circ}$ (approximately 10 $\mathrm{km}$ at the equator). The precipitation considered in the model have been spatially averaged over the study area (see Figure 2). For what concerns the implementation of vaccines in 2015, we considered the 167'377 OCV doses that were distributed in the county of Juba [52] during 6 days of a mass vaccination campaign started in July 31, 2015. Additional details about the model setup can be found in [56]. 


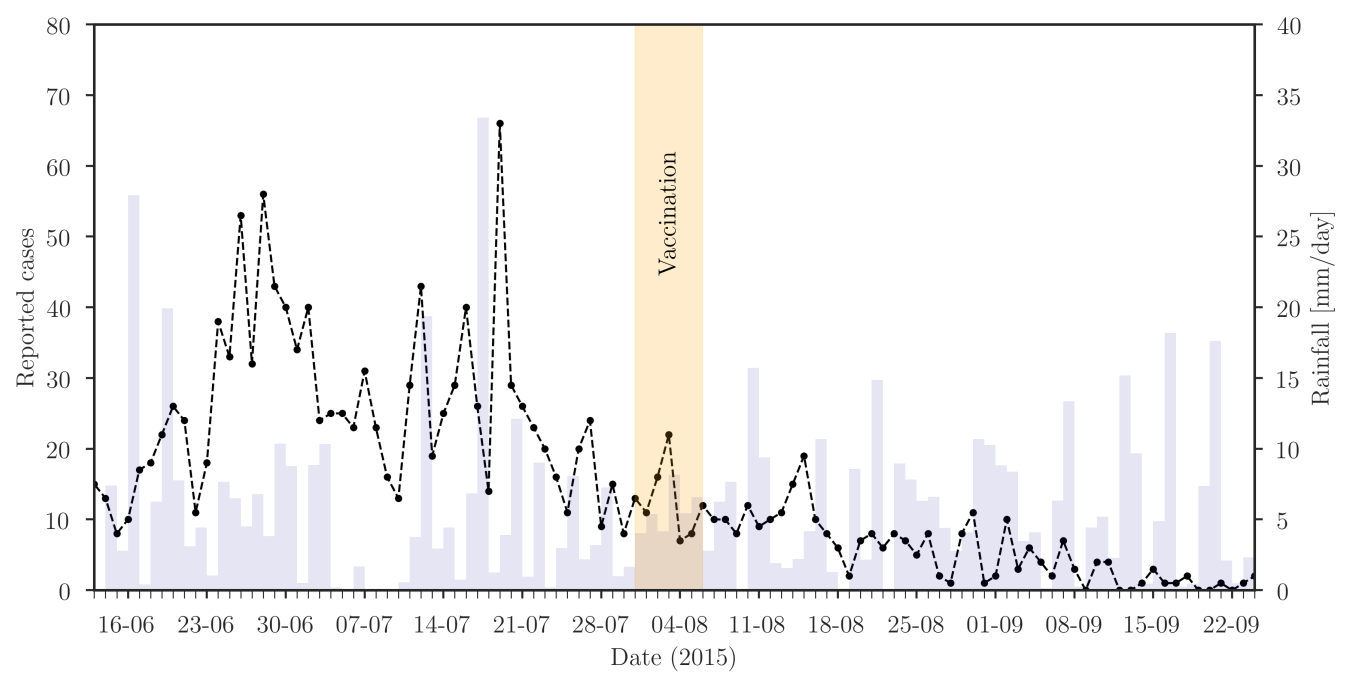

Figure 2: Reported cholera cases (dots) versus precipitation (bars) during the 2015 epidemic in Juba. The timing of the vaccination campaign is highlighted in yellow.

\subsection{Initial conditions}

The past history of cholera epidemics in South Sudan, and particularly in Juba (see Section S.5), plays an important role in the determination of the size of susceptible and recovered compartments at the beginning of the 2015 epidemic. These two compartments are also largely impacted by the rate of immunity loss, $\rho$, of the recovered individuals, which determines the duration of the stay in the $R$ compartment (from few months to several years), and the probability of asymptomatic infected, $1-\sigma$, which determines how many asymptomatics enter the $R$ compartment (values in literature range between $\sigma=0.5$, meaning one asymptomatic per each symptomatic infected, to less than $\sigma=0.01$, corresponding to more than 99 asymptomatic infected per each symptomatic infected [57]. The initial conditions must therefore be estimated for each parameter set considered during calibration. 
To take into account the uncertainty associated with the past epidemics and vaccination campaigns, the initial number of temporary immune individuals, $R_{0}$, in April 2014 is calibrated for each model.

The detailed daily data of suspected cases during 2014 is used to estimate the associated number of recovered individuals. These undergo an exponential decay with average time of immunity loss $1 / \rho$ (similarly to what done in [40]), thus obtaining a consistent estimate of the recovered in June 2016. Simulations are then initialized on the $5^{t} h$ of June 2015 considering two exposed individuals, two infected, and the associated steady-state bacteria concentration.

\section{Results}

\subsection{Selection of rainfall effects on transmission pathways}

The summary statistics of the deterministic and stochastic models considered in the study are given in Table 2. Overall, the stochastic models outperform their deterministic counterparts for all model structures by $\approx 40$ log-likelihood units. Both model types agree in the significance of rainfall in explaining the time series of daily reported cases, in particular through the increased exposure pathway, although the specific ordering of the models differs between model types. Indeed, the BFs for the deterministic models suggest a strong support for model MEC, followed by model ME $\left(B F_{M E, M E H}^{-1}=0.16\right)$, with very little support for all other models $\left(B F_{,, M E H}^{-1}<10^{-2}\right.$ for all other models than ME). For the stochastic model, the BFs estimated with the BIC suggest the strongest support for model ME, with the basic SIRB model coming in second with 5 times less evidence $\left(B F_{M N, M E}^{-1} \approx 0.15\right)$. When 
considering only the BIC, model ME ranks first for both the deterministic and the stochastic formulations. Interestingly, all models that include human-to-human transmission present smaller or equal log-likelihoods than their counterparts with only the bacteria compartment, which suggests that the data does not support both environmental and human-to-human transmission within the set of the models we considered here.

The results of the nested LR-tests confirm the statistical significance of including rainfall in the cholera transmission models, with the effect on exposure better supported by the data in both model types than the effect on contamination. In the deterministic case, the extension of the basic SIRB (model MN) with rainfall effects were significant for all direct comparisons (Fig. 3 A). The addition of human-to-human transmission was not significant mostly due to the above-mentioned lower estimate of the log-likelihood in these models. When considering only a single effect of rainfall (either increasing exposure or contamination), ME outperforms MC in terms of likelihood for the same number of parameters. Interestingly, the inclusion of rainfall-induced contamination in model $\mathrm{ME}$ is rejected due to the very limited increase of the estimated log-likelihood of MEC, in contrast with the BFs favouring the latter. Model ME is thus the one retained by the LR-tests in the deterministic set of models. In the case of the stochastic models, the LR-tests also highlight the importance of the effect of rainfall on exposure rather than on contamination (Fig. $3 \mathrm{~B}$ ). In fact, the much stronger performance of $\mathrm{MN}$ in comparison with its deterministic counterpart relative to all other model structures imposes a stronger condition for retaining additional transmission processes. Indeed, both models $\mathbf{M C}$ and $\mathbf{M C H}$ were 


\begin{tabular}{|c|c|c|c|c|c|c|c|c|}
\hline \multirow[b]{2}{*}{ Model } & \multicolumn{4}{|c|}{ Deterministic } & \multicolumn{4}{|c|}{ Stochastic } \\
\hline & $n$ & $\hat{\ell}$ & $\mathrm{BIC}$ & $B F^{-1}$ & $n$ & $\begin{array}{c}\hat{\ell} \\
\text { (s.e.) }\end{array}$ & $\mathrm{BIC}$ & $B F^{-1}$ \\
\hline $\mathrm{MN}$ & 7 & -368.62 & 770.27 & $3.1 \mathrm{E}-05$ & 8 & $\begin{array}{c}-326.45 \\
(0.105)\end{array}$ & 690.65 & $1.5 \mathrm{E}-01$ \\
\hline MNH & 8 & -368.95 & 775.64 & $1.1 \mathrm{E}-09$ & 9 & $\begin{array}{c}-327.52 \\
(0.052)\end{array}$ & 697.51 & 4.7E-03 \\
\hline MC & 9 & -358.32 & 759.11 & $5.5 \mathrm{E}-03$ & 10 & $\begin{array}{c}-323.50 \\
(0.037)\end{array}$ & 696.01 & $2.5 \mathrm{E}-02$ \\
\hline $\mathrm{MCH}$ & 10 & -359.06 & 765.30 & $1.7 \mathrm{E}-04$ & 11 & $\begin{array}{c}-324.89 \\
(0.041)\end{array}$ & 701.68 & $5.9 \mathrm{E}-04$ \\
\hline $\mathrm{ME}$ & 9 & -356.96 & 756.40 & $1.6 \mathrm{E}-01$ & 10 & $\begin{array}{c}\mathbf{- 3 1 9 . 8 1} \\
(0.035)\end{array}$ & 687.38 & 1 \\
\hline MEH & 10 & -358.06 & 763.30 & $6.3 \mathrm{E}-04$ & 11 & $\begin{array}{c}-320.64 \\
(0.030)\end{array}$ & 693.18 & $4.1 \mathrm{E}-02$ \\
\hline MEC & 11 & -356.87 & 765.64 & 1 & 12 & $\begin{array}{c}-320.17 \\
(0.031)\end{array}$ & 696.96 & $6.2 \mathrm{E}-03$ \\
\hline MECH & 12 & -357.55 & 771.73 & $2.4 \mathrm{E}-06$ & 13 & $\begin{array}{c}-320.38 \\
(0.024)\end{array}$ & 702.09 & $4.8 \mathrm{E}-04$ \\
\hline
\end{tabular}

Table 2: Model comparison statistics. We report for each model its number of parameters $n$, the associated estimated log-likelihood $\hat{\ell}$ (and its Monte Carlo standard error for the stochastic model), and the inverse of the Bayes Factor $\left(B F^{-1}\right)$ with respect to the model with the largest evidence. The BFs for the deterministic models were computed directly from the parameters posteriors, whereas for the stochastic models they were estimated with the Bayesian Information Criterion (BIC) as $B F_{i} \approx e^{\frac{1}{2}\left(B I C_{i}-B I C_{m i n}\right)}$. The BIC for the deterministic models was computed using the maximum log-likelihood value visited with the MCMC algorithm across chains. Best values in each column are indicated in bold. 
rejected when compared to $\mathbf{M N}$, thus only models with rainfall-driven exposure were retained. As in the deterministic case, model $\mathbf{M E}$ is the one finally retained due to the lack of significance of the inclusion of additional transmission processes. We here note that the conclusion based on the LRtests for the deterministic models should be taken with caution because the MCMC algorithm used for calibration does not directly aim at maximizing the likelihood, but rather at sampling from the posterior distribution of the parameters given the data and the model. Moreover, the best likelihood visited by the chains when sampling the posteriors that we here use in the LR-tests is not a formal estimate of the models' likelihood. However, the fact that the LR-tests applied to both model types agree with the selection of ME supports their use in both cases.

Both statistical methods for model comparison therefore agree about the importance of the effect of intra-seasonal rainfall on the exposure to transmission during the 2015 cholera epidemic in Juba. For the deterministic type of models the BFs suggest a stronger support for model MEC, and the LR-tests for ME, whereas for the stochastic models both the BIC-based estimates of BFs and the LR-tests favor ME.

\subsection{Intra-seasonal rainfall events and the 2015 Juba epidemic}

The comparison between the estimated output cases computed by the basic SIRB model (MN) and the most significant rainfall-based processes (MEC and ME for the deterministic and stochastic types, respectively) highlight the importance of rainfall in retrieving the second epidemiological peak (Figure 4). Both deterministic and stochastic SIRB fit well the general trend of the data, but they clearly underestimate the large number of 


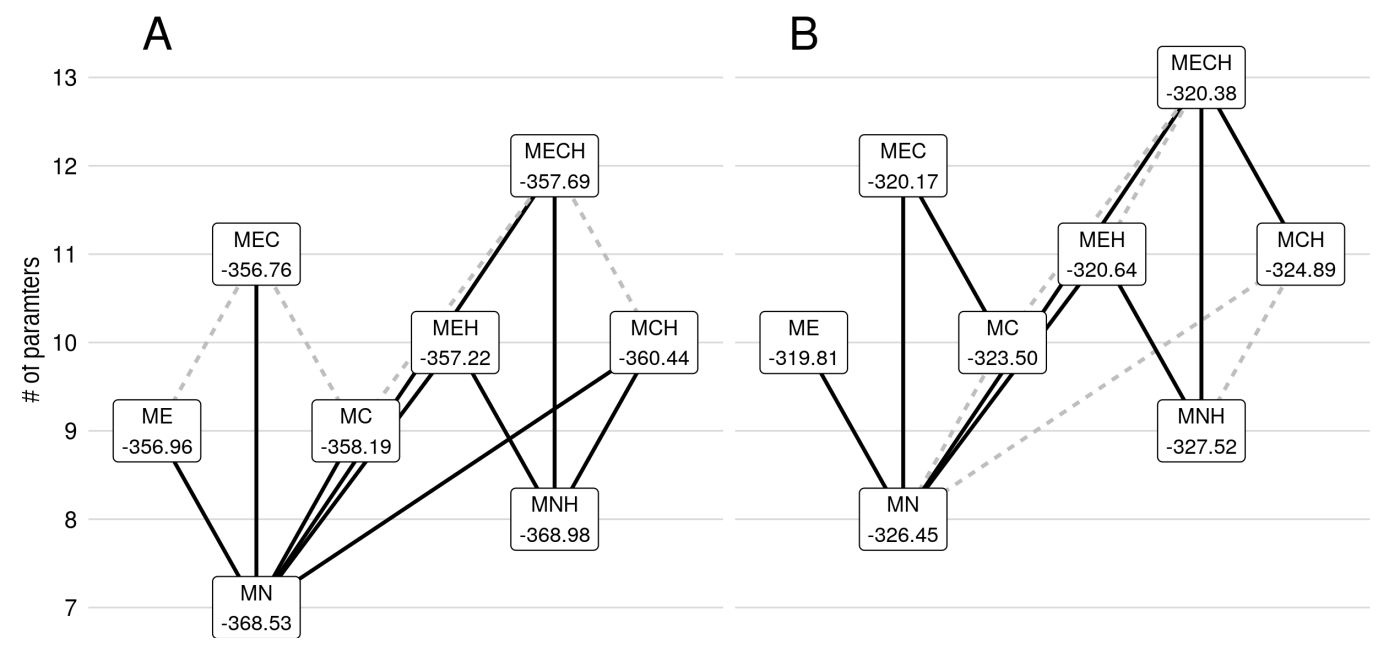

Figure 3: Likelihood ratio tests of model nesting. The LL-tests were computed for each nested pair of models $\left\{\mathcal{M}_{0}, \mathcal{M}_{1}\right\}$, with parameter vectors $\boldsymbol{\theta}^{0}, \boldsymbol{\theta}^{1}$, for which at least on of the parameters that is not null is $\boldsymbol{\theta}^{1}$ is equal to 0 in $\boldsymbol{\theta}^{0}$. Each model is labeled with its associated estimated maximum log-likelihood value, $\hat{\ell}$, for the deterministic (A) and the stochastic (B) models, and linked based on whether the likelihood ratio is significantly (full black lines) or not (dashed gray lines) at the $5 \%$ level. The absence of lines indicates a lower $\hat{\ell}$ for the more complex model. 
reported cases on the 19th of July (65 cases). Instead, the more complex models ME and MEC follow the SIRB dynamics and then are forced by the precipitation occurred in the 18th of July $(33 \mathrm{~mm} / \mathrm{d})$ toward the epidemiological peak.

Model calibration results suggest that precipitations with smaller intensities did not have a strong impact on cholera transmission during the 2015 epidemic in Juba. Indeed, the exponents $\alpha_{\mathcal{C}}$ and $\alpha_{\mathcal{E}}$ were found to be systematically larger than 1 (as shown by posteriors of the deterministic models in Figure S.2 and the Monte Carlo confidence intervals for the stochastic ME in Figure S.4 of the SI). Thus, in the considered epidemic, the nonlinear function used to account for rainfall in the model (eq. 10) helps isolating the contribution of the largest rainfall.

The best measures of fit computed for the stochastic ME (see Table 2) are thus explained by a larger sensitivity to precipitation, which causes the match between the mean of the simulated cases and the data during the second peak.

Comparing the two model types, stochastic results have a larger $95 \%$ confidence interval, which better encompass most of the data. In particular, both epidemiological peaks are well captured by the stochastic models, while the deterministic results systematically underestimate them. Two factors contribute to this result: the intrinsic stochastic nature of the model, that requires the simulation of various model runs for the same set of parameters, and the noise that necessarily perturbs the force of the infection yielding an overdispersion in infections. The standard deviation of such (assumed white) noise is estimated in each stochastic model, and it is interesting to note that 


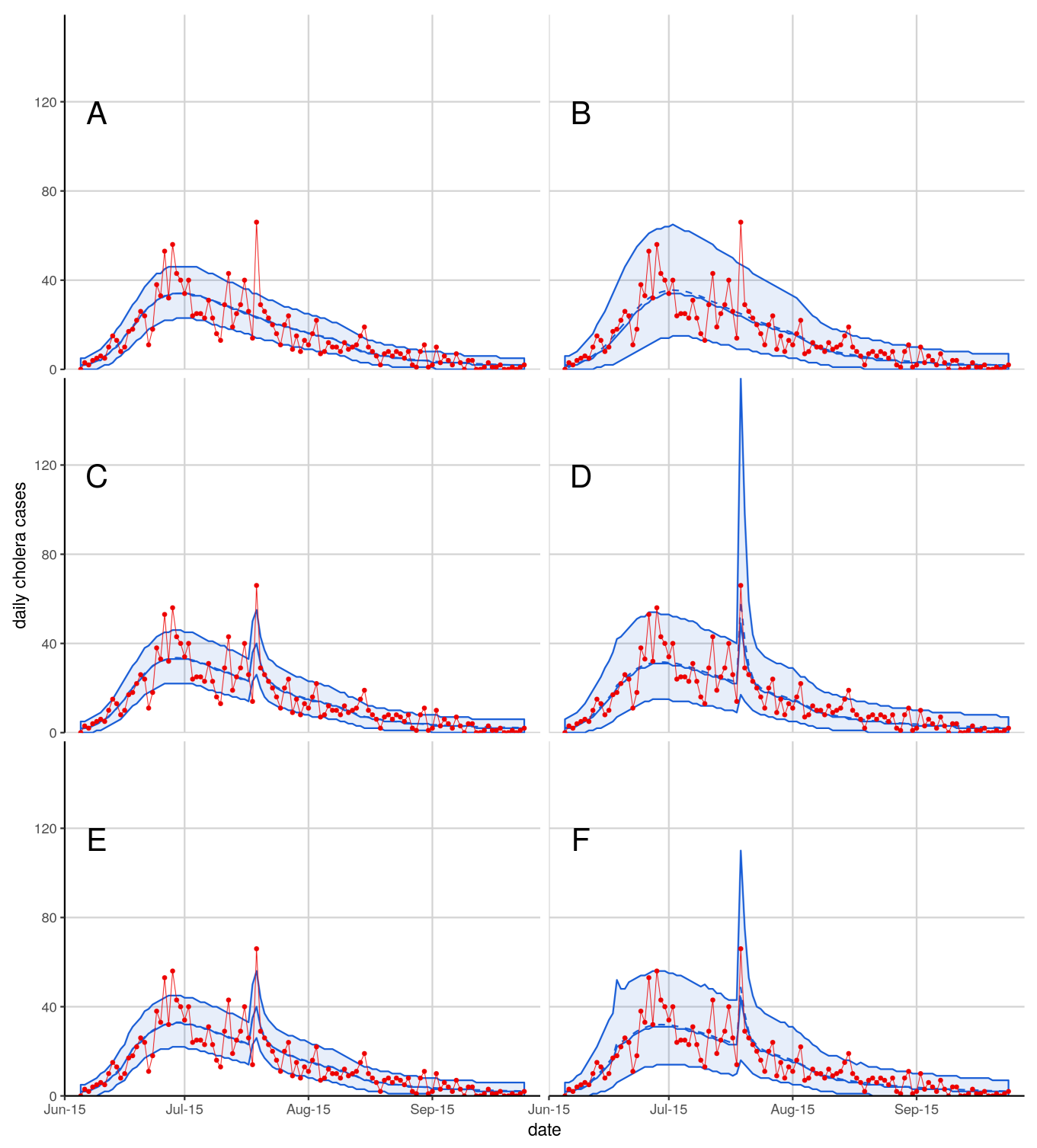

Figure 4: Simulations of the MN (A-B), ME (C-D) and MEC (E-F) models. Simulations for the deterministic versions (A,C,E) are given by the mean (blue dashed line), median (blue full line) and 95\% simulation envelops (blue ribbon) of 100 simulations of the measurement model for each trajectory from 100 samples from the posteriors of model parameters against reported daily cholera cases (red line and dots). Simulations from the stochastic models (B, D, F) are given for 10'000 simulations of the stochastic process and measurement models. 
the MLE obtained for ME is slightly smaller than in MN (0.028 versus 0.022), highlighting again that the data are retrieved with a lower uncertainty when rainfall is included in the model. This is evident in Figure 4, where the width of the $95 \%$ confidence interval of models ME and MEC is smaller with respect to $\mathrm{MN}$.

Finally, despite having different BFs, the deterministic models ME and MEC are qualitatively similar in terms of output response, indicating that the recorded changes in the log-likelihood function do not correspond to qualitative changes in the output.

\section{Discussion and conclusions}

In this study we developed a general mechanistic SIRB-based epidemiological model to evaluate the relevance of rainfall in the amplification of cholera transmission, focusing on the 2015 Juba outbreak. Two rainfall-based transmission processes were compared: the direct increase of the exposure to the contaminated water (model ME) following [24], and the increase of water contamination by flooded open defecation sites (model MC) following [2]. In addition, we also considered human-to-human transmission (models' name with $\mathbf{H})$.

Regarding the epidemiological model, this study introduced two innovations with respect to previous modeling attempts of cholera epidemics (see, e.g., $[43,40]$. First, the focus on daily incidence data, as opposed to weekly epidemiological reports commonly used in modelling works, motivated the introduction of a compartment of exposed individuals (eq. 1) to account for the incubation period of the disease and, thus, the lag between the possibly 
rainfall-driven infection process and the manifestation of the symptoms resulting in the timeseries of daily reported cases at our disposal. Second, a non-linear version of rainfall driver, in the form of a power-law controlled by a single parameter, was introduced to generalize the previous linear dependence. Such formulation has the flexibility to either emphasize the impact of the largest rainfall events, or give equal weight to all non-zero rainfall intensities.

All model assumptions were compared for both deterministic and stochastic model's types, in order to draw more general conclusions. The statistics and tests used to compare the model results (Table 2 and Figure 3) supported the significance of rainfall effects during the 2015 epidemic in Juba. In fact, results showed that for both model types there exists a significant positive effect of including rainfall drivers, in particular because standard SIRB models were not able to reproduce the second epidemiological peak of reported cases occurred in July during the recession period. All models considering rainfall, instead, showed an increase of the number of cases in correspondence of the second epidemiological peak, which was due to the large rainfall rates occurred during the previous days (Fig. 4). This difference in the simulated responses of models considering (or not) rainfall lead to stronger support for rainfall-based models. Due to the small variations among the likelihoods of rainfall-based models, however, (Table 2), it is not straightforward to draw conclusions on the best way to include the rainfall effect. Models with the minimum BIC were those considering the increase in exposure (model ME) for both the stochastic and the deterministic model types. For the deterministic models, the computation of the Bayes Factors 
(BFs), which should provide a direct estimation of the model probability, suggested the selection of the model combining exposure and contamination processes (model MEC). However, this information criterion might be unstable due to numerical issues and oscillations in the MCMC used for calibration [58]. By considering the fact that the models' outputs were similar for MEC and ME (4), we advise to select the approach endowed with less parameters, in this case ME, as indicated by the BIC. Note that the inclusion of human-to-human transmission was not statistically relevant in this modeling exercise.

The comparison between the likelihoods of the two models' types (deterministic and stochastic) showed that considering the stochasticity of the processes improves the model results (Table 2). This suggests that also deterministic models should include a stochastic term in the computation of the force of infection (eq. 9), which might increase the flexibility of the outputs.

Several limitations should be considered when analyzing the present results. The calibration exercise attempted in this study considered daily rainfall and cholera reported cases, which are characterized by significant random fluctuations that might partly cloud the description of the underlying infection processes. Small random delays in reporting could change the infection curve and thus the effect of rainfall. This issue was partially addressed by considering the exposed compartment (1) for simulation of the incubation period, and unknown reporting rate $\epsilon$ for the observed cases.

Here, we strove to reproduce the epidemic by modelling epidemiological transmission processes. While we took into account non-linear rainfall effects 
and possible over-reporting, we did not consider human mobility effect [59, $60,61,23]$, which could help modeling the arrival of infected individual. Moreover, in our model asymptomatic infected individuals did not contribute to the bacterial concentration in the environment, while they might impact the infection cycle due to the presence of bacteria in their feces. From a modeling viewpoint, these unaccounted processes were compensated by the calibration procedure, at the loss of predictive power.

The prior bounds to be assigned to parameters are typically wide [62] because the rates governing transmission processes are highly dependent on the specific epidemiological context, so that somewhat contradictory values had been estimated in literature. These considerations, together with the intrinsic noise affecting recorded cases, underlie the possibility that some of the model parameter might be unidentifiable [63], in the sense that different parameter combinations would yield the same model output (also called equifinality). The exploration of the posterior parameter distribution using an MCMC approach allowed us to evince the possible correlation among parameters that were well identified by the data, with the main risk of the algorithm getting trapped in a local minimum of the fitness landscape (the distribution of parameters). The posterior probability distributions of the model parameters (see SI, Section S4) are associated with the model uncertainty, and were here explored by the chains of the MCMC calibration.

The lack of available data prevented us to include the effects of the overall efforts towards WaSH improvement in this study. This assumption is reasonable in the case at hand, however, given the short time-frame of the study. Despite these limitations, our model comparison using both a deterministic 
and a stochastic model gave coherent results. The agreement of the two modeling types strengthened our results regarding the importance of rainfall patterns to significantly affect the development of cholera cases in time.

Overall, the findings of the study are consistent with the lessons learned in South Sudan with most of the transmission starting with the onset of the rainy season. In 2016 and 2017, cases in the dry season were observed and associated to the overexploitation of scarce water resources by nomadic herdsmen (cattle camps). This suggests that, as already observed, a general assessment of the relationship between precipitation and general waterborne or water-based disease infections is far from obvious and surely case-dependent. It has been argued, for example, that in the domain of water-based parasitic infections (see e.g. [64, 8]) rainfall could not only boost disease transmission (especially in dry climates where it is a key driver of habitat formation for possible intermediate hosts) but also reduce it substantially, e.g. by increasing water flow (which in turn decreases habitat suitability for both the intermediate and the human hosts). Rainfall patterns may also drastically affect human activities related to water contacts, thus potentially altering exposure and transmission risk [65]. To that end, a hydrology-driven assessment cannot ignore certain characteristics, in particular the ephemeral or permanent nature of the waterways fostering contacts among pathogens and hosts [66]. Also, temporal fluctuations of rainfall patterns may be particularly important in determining the seasonality of transmission [67, 68, 69, 66].

Subsuming the results obtained by this major computational exploration focused on the analysis of Juba's 2015 epidemics, we conclude that rainfall patterns are fundamental drivers for epidemic cholera models, whether de- 
terministic or stochastic, not only to capture seasonal trends, but also to describe short-term fluctuations in the number of reported cases.

\section{Acknowledgements}

JL, JPS, DP, and AR acknowledge funds provided by the Swiss National Science Foundation (www.snf.ch), via the projects: "Dynamics and controls of large-scale cholera outbreaks" (DYCHO CR23I2 138104) and "Optimal control of intervention strategies for waterborne disease epidemics" (200021172578). The authors wish to thank Andrew Azman, Johns Hopkins University, for insightful discussions on data collection and model inputs.

\section{References}

[1] J. Snow, On the Mode of Communication of Cholera, John Churchill, 1855.

[2] A. Rinaldo, E. Bertuzzo, L. Mari, L. Righetto, M. Blokesch, M. Gatto, R. Casagrandi, M. Murray, S. Vesenbeckh, I. Rodriguez-Iturbe, Reassessment of the 2010-2011 Haiti cholera outbreak and rainfall-driven multiseason projections, Proceedings of the National Academy of Sciences USA 109 (17) (2012) 6602-6607.

[3] E. Boelee, Y. Mekonnen, J. N. Poda, M. McCartney, P. Checchi, S. Kibret, F. Hagos, H. Laamrami, Options for water storage and rainwater harvesting to improve health and resilience against climate change in Africa, Regional Environmental Change 13 (2013) 509-519. 
[4] F. Finger, T. Genolet, L. Mari, G. C. de Magny, N. M. Manga, A. Rinaldo, E. Bertuzzo, Mobile phone data highlights the role of mass gatherings in the spreading of cholera outbreaks, Proceedings of the National Academy of Science USA 113 (23) (2016) 6421-6426.

[5] J. D. Sugimoto, A. A. Koepke, E. E. Kenah, M. Halloran, F. Chowdhury, et al., Household Transmission of Vibrio cholerae in Bangladesh, PLoS Neglected Tropical Diseases 8 (2014) e3314.

[6] Q. Bi, A. S. Azman, S. M. Satter, A. I. Khan, D. Ahmed, et al., Microscale spatial clustering of cholera risk factors in urban Bangladesh, PLoS Neglected Tropical Diseases 10 (2016) e0004400.

[7] J. Lessler, H. Salje, M. Grabowski, D. Cummings, Measuring spatial dependence for infectious disease, Epidemiology 11(5) (2016) e0155249.

[8] A. Rinaldo, E. Bertuzzo, L. Mari, M. Blokesch, M. Gatto, Modeling key drivers of cholera transmission dynamics provides new perspectives for parasitology, Trends in Parasitology (2017) 113doi:10.1016/j.pt.2017.04.002.

[9] R. Colwell, Global climate and infectious disease: the cholera paradigm, Science 274 (1996) 2025-2031.

[10] M. Pascual, X. Rodo, S. Ellner, R. Colwell, M. Bouma, Cholera dynamics and El Nino-Southern oscillation, Science 289 (2000) 1766-1769.

[11] M. Hashizume, B. Armstrong, S. Hajat, Y. Wagatsuma, A. S. Faruque, T. Hayashi, D. A. Sack, The effect of rainfall on the incidence of cholera in Bangladesh, Epidemiology 19 (1) (2008) 103-110. 
[12] G. Constantin de Magny, W. Thiaw, V. Kumar, N. Manga, B. M. Diop, L. Gueye, M. Kamara, B. Roche, R. Murtgudde, R. R. Colwell, Cholera outbreak in Senegal in 2005: Was climate a factor?, PLoS ONE 7 (2012) e44577.

[13] M. Hashizume, L. F. Chaves, A. S. G. Faruque, A differential effect of Indian Ocean Dipole and El Niño on cholera dynamics in Bangladesh, PLoS ONE 8 (2013) e60001.

[14] X. Rodó, M. Pascual, F. J. Doblas-Reyes, A. Gershunov, D. A. Stone, F. Giorgi, P. J. Hudson, J. Kinter, M.-A. Rodríguez-Arias, N. C. Stenseth, D. Alonso, J. García-Serrano, A. P. Dobson, Climate change and infectious diseases: Can we meet the needs for better prediction?, Climatic Change 118 (2013) 625-40.

[15] I. J. Ramírez, S. C. Grady, El Niño, climate, and cholera associations in Piura, Peru, 1991-2001: A wavelet analysis, EcoHealth 13 (2016) 83-99.

[16] L. Vezzulli, C. Grande, P. C. Reid, P. Hélaouët, M. Edwards, M. G. Höfle, I. Brettar, R. R. Colwell, C. Pruzzo, Climate influence on Vibrio and associated human diseases during the past half-century in the coastal North Atlantic, Proceedings of the National Academy of Sciences USA 113 (2016) 5062-5071.

[17] R. C. Reiner, A. A. King, M. Emch, M. Yunus, A. Faruque, M. Pascual, Highly localized sensitivity to climate forcing drives endemic cholera in a megacity, Proceedings of the National Academy of Sciences 109 (6) (2012) 2033-2036. 
[18] C. Baker-Austin, J. A. Trinanes, N. G. H. Taylor, R. Hartnell, A. Siitonen, J. Martinez-Urtaza, Emerging vibrio risk at high latitudes in response to ocean warming, Nature Climate Change 3 (2013) 73-77.

[19] L. Vezzulli, R. Colwell, C. Pruzzo, Ocean warming and spread of pathogenic vibrios in the aquatic environment, Microbial Ecology 65 (2013) 817-825.

[20] B. Cash, X. Rodó, M. Emch, M. Yunus, A. Faruque, M. Pascual, Cholera and shigellosis: Different epidemiology but similar responses to climate variability, PLoS ONE 9 (2014) e107223.

[21] L. E. Escobar, S. J. Ryan, A. M. Stewart-Ibarra, J. L. Finkelstein, C. A. King, H. Qiao, M. E. Polhemus, A global map of suitability for coastal Vibrio cholerae under current and future climate conditions, Acta Tropica 149 (2015) 202-211.

[22] L. Vezzulli, E. Pezzati, I. Brettar, M. Höfle, C. Pruzzo, Effects of global warming on Vibrio ecology, Microbiology Spectrum 3 (2015) 0004-2014.

[23] J. Perez-Saez, A. A. King, A. Rinaldo, M. Yunus, A. S. Faruque, M. Pascual, Climate-driven endemic cholera is modulated by human mobility in a megacity, Advances in Water Resources 108 (2017) 367 - 376. doi:10.1016/j.advwatres.2016.11.013.

[24] M. Eisenberg, G. Kujbida, A. Tuite, D. Fisman, J. Tien, Examining rainfall and cholera dynamics in Haiti using statistical and dynamic modeling approaches, Epidemics 5 (4) (2013) 197-207. 
[25] T. Baracchini, A. A. King, M. J. Bouma, X. Rodó, E. Bertuzzo, M. Pascual, Seasonality in cholera dynamics: A rainfall-driven model explains the wide range of patterns in endemic areas, Advances in Water Resources 108 (2016) 357-366.

[26] D. Ruiz-Moreno, M. Pascual, M. Bouma, A. Dobson, B. Cash, Cholera seasonality in madras (1901-1940): Dual role for rainfall in endemic and epidemic regions, EcoHealth 4 (2007) 52-62.

[27] E. Lipp, A. Huq, R. Colwell, Effects of global climate on infectious disease: The cholera model, Clinical Microbiology Reviews 15 (4) (2002) $757-770$.

[28] S. M. Faruque, M. J. Islam, Q. S. Ahmad, A. S. G. Faruque, D. A. Sack, G. B. Nair, J. J. Mekalanos, Self-limiting nature of seasonal cholera epidemics: Role of host-mediated amplification of phage, Proceedings of the National Academy of Sciences USA 102 (2005) 6119-6124.

[29] V. R. Hill, N. Cohen, A. M. Kahler, J. L. Jones, C. A. Bopp, N. Marano, C. L. Tarr, N. M. Garrett, J. Boncy, A. Henry, G. A. Gomez, M. Wellman, M. Curtis, M. M. Freeman, M. Turnsek, R. A. Benner, Jr., G. Dahourou, D. Espey, A. DePaola, J. W. Tappero, T. Handzel, R. V. Tauxe, Toxigenic Vibrio cholerae O1 in water and seafood, Haiti, Emerging Infectious Diseases 17 (11) (2011) 2147-2150.

[30] S. Rebaudet, B. Sudre, B. Faucher, R. Piarroux, Environmental determinants of cholera outbreaks in inland Africa: a systematic review of 
main transmission foci and propagation routes, The Journal of Infectious Diseases 208 (2013) S46-S54.

[31] M. Emch, C. Feldacker, M. S. Islam, M. Ali, Seasonality of cholera from 1974 to 2005: a review of global patterns, International Journal of Health Geographics 7 (1) (2008) 31-39. doi:10.1186/1476-072X-7-31.

[32] G. Constantin de Magny, R. Murtugudde, M. R. P. Sapiano, A. Nizam, C. W. Brown, A. J. Busalacchi, M. Yunus, G. B. Nair, A. I. Gil, C. F. Lanata, J. Calkins, B. Manna, K. Rajendran, M. K. Bhattacharya, A. Huq, R. B. Sack, R. R. Colwell, Environmental signatures associated with cholera epidemics, Proceedings of the National Academy of Sciences USA 105 (2008) 17676-17681.

[33] S. Rebaudet, B. Sudre, B. Faucher, R. Piarroux, Cholera in coastal africa: a systematic review of its heterogeneous environmental determinants, The Journal of Infectious Diseases 208 (2013) S98-S106.

[34] A. Jutla, A. Akanda, A. Huq, A. Faruque, R. Colwell, S. Islam, A water marker monitored by satellites to predict seasonal endemic cholera, Remote Sensing Letters 4 (8) (2013) 822-831.

[35] J. Gaudart, S. Rebaudet, R. Barrais, J. Boncy, B. Faucher, M. Piarroux, R. Magloire, G. Thimothe, R. Piarroux, Spatio-temporal dynamics of cholera during the first year of the epidemic in Haiti, PLoS Neglected Tropical Diseases 7 (4) (2013) e2145.

[36] R. R. Frerichs, P. S. Keim, R. Barrais, R. Piarroux, Nepalese origin 
of cholera epidemic in Haiti, Clinical Microbiology and Infection 18 (6) (2012) E158-E163.

[37] A. Kirpich, T. Weppelmann, Y. Yang, A. Ali, J. Morris Jr., I. Longini, Cholera transmission in ouest department of haiti: Dynamic modeling and the future of the epidemic, PLoS Neglected Tropical Diseases 9 (10) (2015) e0004153. doi:10.1371/journal.pntd.0004153.

[38] ERCC ECHO, Haiti - Hurricane Matthew - Damage assessment and ECHO Response, Emergency Response Coordination Centre (ERCC), European Commission, Humanitarian Aid and Civil Protection (2016 (accessed on 2017-14-04)).

URL http: //erccportal.jrc.ec.europa.eu/getdailymap/docId/1774

[39] A. Camacho, D. Pasetto, F. Finger, E. Bertuzzo, S. Cohuet, F. Grandesso, E. Lynch, F. Luquero, Prediction of cholera dynamics in Haiti following the passage of Hurricane Matthew, Tech. rep., Epicentre, Paris, France (2016).

[40] D. Pasetto, F. Finger, A. Rinaldo, E. Bertuzzo, Real-time projections of cholera outbreaks through data assimilation and rainfall forecasting, Advances in Water Resources 108 (2017) 345-356.

[41] R. Khan, R. Anwar, S. Akanda, M. D. McDonald, A. Huq, A. Jutla, R. Colwell, Assessment of risk of cholera in haiti following hurricane matthew, The American Journal of Tropical Medicine and Hygiene 97 (3) (2017) 896-903. doi:10.4269/ajtmh.17-0048. 
[42] R. Piarroux, R. Barrais, B. Faucher, R. Haus, M. Piarroux, J. Gaudart, R. Magloire, D. Raoult, Understanding the cholera epidemic, Haiti, Emerging Infectious Diseases 17 (2011) 1161-1168.

[43] E. Bertuzzo, F. Finger, L. Mari, M. Gatto, A. Rinaldo, On the probability of extinction of the Haiti cholera epidemic, Stochastic Environmental Research and Risk Assessment 30 (2016) 2043-2055. doi:10.1007/s00477-014-0906-3.

[44] P. Adams, Haiti prepares for cholera vaccination but concerns remain, The Lancet 379 (2012) 16.

[45] M. Periago, T. Frieden, J. Tappero, K. De Cock, B. Aasen, J. Andrus, Elimination of cholera transmission in haiti and the dominican republic, The Lancet 379 (2012) E12-E13.

[46] P. Adams, Cholera in Haiti takes a turn for the worse, The Lancet 381 (9874) (2013) 1264.

[47] C. Codeço, Endemic and epidemic dynamics of cholera: the role of the aquatic reservoir, BMC Infectious Diseases 1 (1).

[48] A. S. Azman, K. E. Rudolph, D. A. Cummings, J. Lessler, The incubation period of cholera: A systematic review, Journal of Infection 66 (5) (2013) 432 - 438. doi:10.1016/j.jinf.2012.11.013.

[49] C. Bretó, D. He, E. L. Ionides, A. A. King, et al., Time series analysis via mechanistic models, The Annals of Applied Statistics 3 (1) (2009) 319-348. 
[50] A. Abubakar, A. S. Azman, J. Rumunu, I. Ciglenecki, T. Helderman, H. West, J. Lessler, D. A. Sack, S. Martin, W. Perea, D. Legros, F. J. Luquero, The First Use of the Global Oral Cholera Vaccine Emergency Stockpile: Lessons from South Sudan, PLoS Medicine 12 (11) (2015) e1001901. doi:10.1371/journal.pmed.1001901.

[51] A. S. Azman, L. A. Parker, J. Rumunu, F. Tadesse, F. G. et al., Effectiveness of one dose of oral cholera vaccine in response to an outbreak: a case-cohort study, The Lancet Global Health 4 (11) (2016) e856 - e863. doi:10.1016/S2214-109X(16)30211-X.

[52] L. Parker, J. Rumunu, C. Jamet, Y. Kenyi, R. Laku Lino, J. F. Wamala, A. M. Mpairwe, F. J. Ciglenecki, I. Luquero, A. S. Azman, J.-C. Cabrol, Adapting to the global shortage of cholera vaccines: targeted single dose cholera vaccine in response to an outbreak in South Sudan, Lancet Infectious Diseases 201717 (4) (2017) e123e127. doi:10.1016/S14733099(16)30472-8.

[53] SSNBS, Population projections for South Sudan by County 2015 - 2020, Tech. rep., South Sudan National Bureau of Statistics (2015).

[54] CIA, South Sudan, Central Intelligence Agency. Available online., 2015.

[55] IRI/LDEO, http://iridl.ldeo.columbia.edu/, climate Data Library. Available online; accessed on 7-April-2016 (2016).

[56] C. Sciarra, A. Rinaldo, F. Laio, D. Pasetto, Mathematical modeling of cholera epidemics in south sudan, arXiv preprint arXiv:1801.03125. 
[57] I. C.-H. Fung, Cholera transmission dynamic models for public health practitioners, Emerging Themes in Epidemiology 11 (1).

[58] A. E. Raftery, M. A. Newton, J. M. Satagopan, P. N. Krivitsky, Estimating the integrated likelihood via posterior simulation using the harmonic mean identity, in: Bayesian Statistics, Oxford University Press, New York, 2007, pp. 1-45.

[59] M. Gatto, L. Mari, E. Bertuzzo, R. Casagrandi, L. Righetto, I. Rodriguez-Iturbe, A. Rinaldo, Generalized reproduction numbers and the prediction of patterns in waterborne disease, Proceedings of the National Academy of Sciences USA 48 (2012) 19703-19708.

[60] E. Bertuzzo, R. Casagrandi, M. Gatto, I. Rodriguez-Iturbe, A. Rinaldo, On spatially explicit models of cholera epidemics, Journal of the Royal Society Interface 7 (2010) 321-333.

[61] L. Mari, E. Bertuzzo, F. Finger, R. Casagrandi, M. Gatto, A. Rinaldo, On the predictive ability of mechanistic models for the haitian cholera epidemic, Journal of the Royal Society Interface 12 (104) (2015) 20140840. doi:10.1098/rsif.2014.0840.

[62] O. Akman, M. R. Corby, E. Schaefer, Examination of models for cholera: insights into model comparison methods, Letters in Biomathematics 3 (1) (2016) 93-118. doi:10.1080/23737867.2016.1211495.

[63] M. C. Eisenberg, S. L. Robertson, J. H. Tien, Identifiability and estimation of multiple transmission pathways in cholera and waterborne disease, Journal of theoretical biology 324 (2013) 84-102. 
[64] N. McCreesh, M. Booth, Challenges in predicting the effects of climate change on Schistosoma mansoni and Schistosoma haematobium transmission potential, Trends Parasitol. 29 (2013) 548-555.

[65] Y. Lai, P. Biedermann, U. Ekpo et al., Spatial distribution of schistosomiasis and treatment needs in sub-Saharan Africa: A systematic review and geostatistical analysis, Lancet Infect. Dis. 15 (2015) 927-940.

[66] J. Perez-Saez, T. Mande, N. Ceperley, E. Bertuzzo, L. Mari, M. Gatto, A. Rinaldo, Hydrology and density feedbacks control the ecology of the intermediate hosts of schistosomiasis across habitats in seasonal climates, Proc. Natl. Acad. Sci. USA 113 (2016) 6427-6432.

[67] E. Bertuzzo, L. Mari, L. Righetto, M. Gatto, R. C. andI. RodriguezIturbe, A. Rinaldo, Hydroclimatology of dual-peak annual cholera incidence: Insights from a spatially explicit model, Geophysical Research Letters 39 (2012) L05403.

[68] E. Bertuzzo, L. Mari, L. Righetto, M. Gatto, R. Casagrandi, I. Rodriguez-Iturbe, A. Rinaldo, Prediction of the spatial evolution and effects of control measures for the unfolding Haiti cholera outbreak, Geophys. Res. Lett. 38 (2011) L06403.

[69] N. McCreesh, G. Nikulin, M. Booth, Predicting the effects of climate change on Schistosoma mansoni transmission in eastern Africa, Parasite. Vect. 8 (2015) 4. 